\title{
Editorial
}

\section{Interview with Prof. Dr Richard Casaburi, Presidential Awardee 2020}

\section{Richard Casaburi, PhD, MD}

Professor of Medicine, UCLA School of Medicine Medical Director, Rehabilitation Clinical Trials Center, Lundquist Institute for Biomedical Innovation at Harbor-UCLA Medical Center, Torrance, CA, USA;

Fellow of the European Respiratory Society, the American College of Chest Physicians and the American Association of Cardiovascular and Pulmonary Rehabilitation

Member of the Board of Directors of the COPD Foundation (USA)

"The mission for pulmonary rehabilitation for the next 10 years is three-fold: 1) access,

2) access, and 3) access..."

Prof. Dr Casaburi has an "unusual" track record: he completed his undergraduate degree in electrical engineering and followed with a master's degree and doctorate in biomedical engineering, before completing a post-doctoral fellowship in biomedical engineering at the University of Southern California (Los Angeles, CA, USA). Five years after joining the Dept of Medicine faculty at Harbor-UCLA Medical Center (Torrance, CA, USA), Dr Casaburi returned to school to pursue his medical degree. Later he re-joined the faculty of the Division of Respiratory Medicine at Harbor-UCLA, where he later became Chief. He is currently Associate Chief for Research in the Division of Respiratory and Critical Care

@ERSpublications

Pulmonary rehabilitation has evolved from an "art" to "science" over the past decades thanks to the contributions of Prof Casaburi. His priorities for the future are: 1) access, 2) access, and 3) access! https://bit.ly/3j2aSdr
Physiology and Medicine at Harbor-UCLA Medical Center. He has occupied the Grancell/Burns Chair in the Rehabilitative Sciences at the Lundquist Institute for Biomedical Innovation at Harbor-UCLA Medical Center since 2001. The milestones of his career are summarised in figure 1.

Dr Casaburi established the Rehabilitation Clinical Trials Center in 1999, a clinical research facility dedicated to improving the lives of COPD patients. Dr Casaburi and his group of investigators have completed more than 75 clinical research studies, including participation in three major $\mathrm{NIH}$ multicentre projects. He has published more than 350 papers and accumulated 70000 citations in Google Scholar. With his mentor Prof. Tom Petty he authored the textbook "Principles and Practice of Pulmonary Rehabilitation" [1], which long served as the reference work to the field.

Prof. Casaburi has applied the science of exercise physiology to the practice of pulmonary rehabilitation and hence contributed to the conversion of pulmonary rehabilitation from an "art" to a "science". His studies showed that it was possible to conduct high-intensity exercise training in patients with COPD, even in patients with severe disease. His studies are rigorously designed and shed light on important clinical questions, such as the role of oxygen therapy in pulmonary rehabilitation and the role of testosterone supplementation. He engaged in several large multicentre studies and was among the first to actively collaborate with the pharmaceutical industry to bring exerciserelated end-points to the field of COPD. He is a true
Cite as: Troosters $T$, Casaburi R. Interview with Prof. Dr Richard Casaburi, Presidential Awardee 2020. Breathe 2020; 16: 200249.

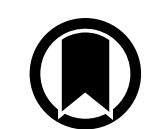

CrossMark 


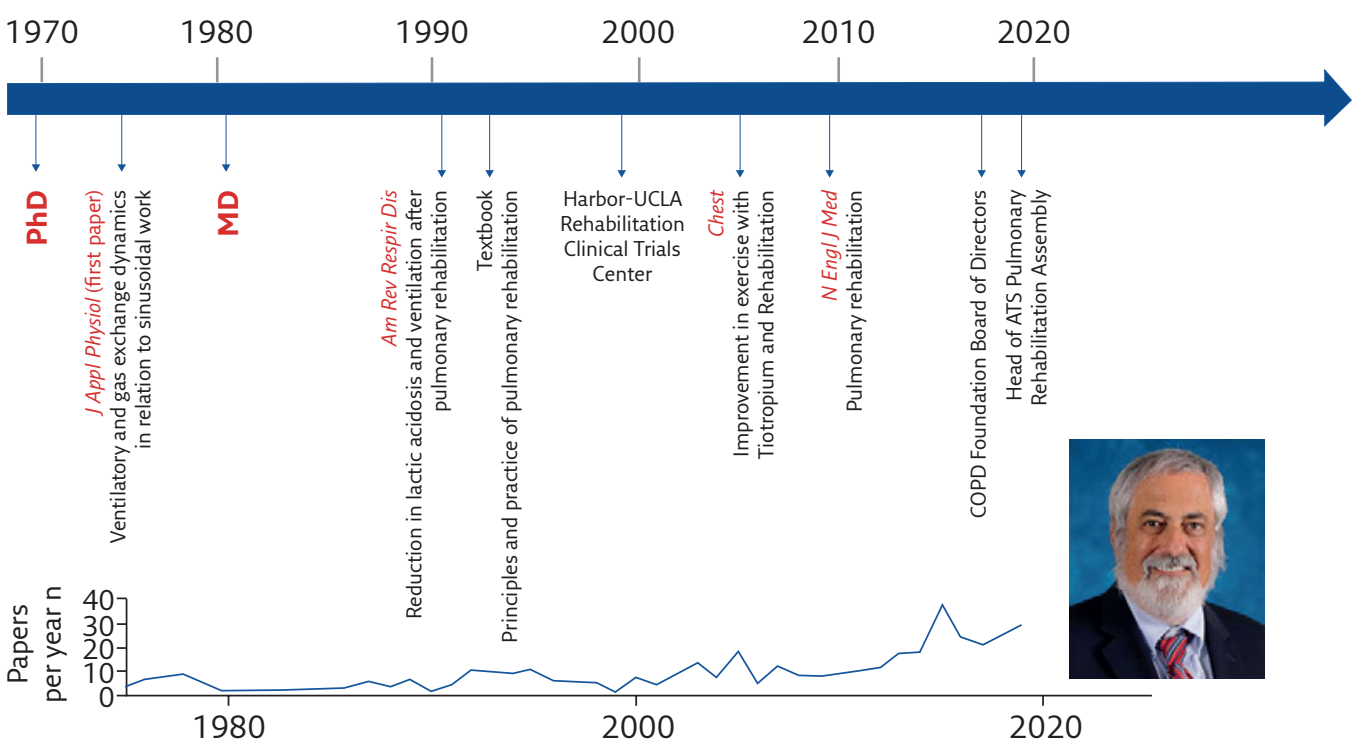

Figure 1 Milestones in the career of Prof. Dr Richard Casaburi. ATS: American Thoracic Society.

advocate for pulmonary rehabilitation and, through his work with the advocacy team of the American Thoracic Society and the COPD Foundation, has kept pulmonary rehabilitation and care for COPD patients on the map in the USA. Recently, with the COPD Foundation, a US-based patient-centred organisation, he co-chaired the COPD Biomarker Qualification Consortium group which is attempting to gain regulatory acceptance for clinically relevant exercise tolerance outcomes for use in COPD clinical trials. Clearly many of these achievements are a team effort, but the contributions of Prof. Casaburi cannot be underestimated.

On 22 September 2020, Thierry Troosters, European Respiratory Society (ERS) 2019-2020 president, conducted an interview with Prof. Casaburi based on a first round of written answers to his questions. The interview is available in full in the supplementary material.

Prof. Casaburi, I'm excited to award you the presidential award at the 30th ERS Congress. I was in kindergarten when you published your first papers in the 1970s. All these years you've published relevant work on the assessment and understanding of exercise intolerance and on exercise training. In recent years, end-points for COPD research have become increasingly patient reported or patient centred. To what extend to you think a good understanding of the principles of exercise limitation can help us understand the impact of meaningful interventions for COPD? Do you think clinicians should know more about exercise principles in order to understand the benefits of the treatments they offer?

Thank you Dr Troosters for this great honour. I'm glad to have the opportunity to reflect on how pulmonary rehabilitation has advanced over the years. In the years after Tom Petty (and others) introduced programmes of pulmonary rehabilitation as one of the therapeutic options for COPD patients, it gained a following but suffered from the impression that it had mainly psychologic benefits for the patient. In a way, the situation was similar to what we have now with many of the home rehabilitation programmes: the mechanisms and magnitude of benefits were unclear. Exercise programmes are a major focus of pulmonary rehabilitation. Understanding the mechanisms by which exercise training benefits the COPD patient lets us rationally design rehabilitative exercise programmes and gauge their success.

One of the many reasons to award this to you is your contribution to the field of pulmonary rehabilitation. You have arguably helped to shape the field and surely you have put the exercise science in this field. Your paper in 1991 [2], perhaps along with that of our friend Francois Maltais [3] proved that exercise training was 
possible in patients with COPD and resulted in significant benefits of patients. We're now 25-30 years later; what are, in your opinion, other milestones that shaped the field of rehabilitation for patients with respiratory conditions?

The story actually begins before 1991. In a 1987 exercise training study performed in healthy subjects [4], we observed reductions in the pulmonary ventilation required for a heavy exercise task. We speculated, at the time, that patients for whom limitations in ventilation are present would benefit from exercise training by this mechanism. In the study you mentioned, performed in collaboration with Italian colleagues, we validated this mechanism, showing that exercise training yielded reduced ventilatory requirements and enhanced exercise tolerance. Prof. Francois Maltais and his group took this further, performing muscle biopsies before and after training, and showed that morphologic and biochemical changes similar to those in healthy subjects were present. Together, these studies (and many others) validated the concept that skeletal muscle dysfunction was widely present in COPD patients, and that it was largely reversible. Among the therapies we have for COPD patients, pulmonary rehabilitation is far and away the most effective in improving the ability to tolerate activities in their everyday life, very often the patient's chief complaint.

Of the subsequent milestones, l'd highlight the realisation that physical activity in everyday life is critically important in the prognosis of the COPD patient [5]. Development of methods to quantitate physical activity and strategies to durably improve it have been among your most productive research focuses, Thierry. In the past decade, we've explored interventions focused on improving physical activity. Traditional programmes of pulmonary rehabilitation often, but not always, result in improvements in physical activity in their daily life. Adding formal behaviour modification techniques, pedometer feedback and maintenance programmes to traditional pulmonary rehabilitation programmes promise to make them more effective.

\section{You bring up two important concepts, relevant to the success of exercise training and rehabilitation. Physiologic improvements and behaviour change. Zooming in on the former, what are the essential requirements for an exercise training programme to be effective in improving physiology?}

This is a difficult question to answer as we're still not completely aware of the underlying molecular mechanisms by which exercise causes the structural and biochemical changes in the exercising muscle. What can be said is that the exercise programme has to involve intensities above that the patient performs in their everyday life and the higher the intensity the better. These are critical concepts, but how to blend these into the most effective programme is not yet fully understood.

Recently, you were involved in a study using "virtual reality" training (combined with or without conventional endurance training) [6]. To what extent do you think these "novel" forms of pulmonary rehabilitation will find a way into the market? Like you, I remain concerned these programmes do not adhere to "exercise training laws" [7], but the success of a programme may depend on the end-points of interest. So let me ask you, with your different hats on (exercise physiologist, clinician, COPD Foundation Board member and regulatory affairs expert), what is in your opinion the preferred primary end-point of a pulmonary rehabilitation trial?

I've had seriously mixed feelings about promoting various alternatives to traditional in-centre programmes. I respect others who are pursuing these alternatives, though, and hope that research featuring well-designed comparative studies will continue to be performed. My personal belief is that, without the in-person individualised attention patients receive from skilled therapists and without the therapeutic interaction among patient participants, remote rehabilitation will be unable to achieve the magnitude of benefits (physiologic and otherwise) seen in traditional programmes. My fear is that, seeing a less expensive alternative, that payors will further disincentivise in-centre programmes, reducing their availability even further.

Recent developments have changed my mind, though. With the coronavirus disease 2019 crisis, in-centre programmes have become nearly unavailable. Remote programmes are an alternative we have to explore and promote. The debate over whether or not they are equivalent (or even superior) can wait until the pandemic is in the rear view mirror.

I certainly agree, so let's fast forward 2 years (to be on the safe 
side), what would you recommend as realistic and decisive endpoints for clinical trials moving forward if you wanted to show equivalence or superiority of "novel" forms of rehabilitation?

You ask hard questions! Certainly physiologic end-points (including exercise tolerance) would be on my list. Another subtle point is that such trials should include a comparator group of patients undergoing in-centre rehabilitation. But even that may not be adequate. In some studies, the in-centre programme has not yielded results we've come to expect from rehabilitation programmes featuring high intensity exercise programmes, these comparisons need to be examined. With good people working together collaboratively, we'll find the answers.

Pulmonary rehabilitation is largely underused around the globe. A recent study in the USA showed that only a few percent of patients are offered the intervention after a severe exacerbation, a moment where pulmonary rehabilitation may be lifesaving. Why is it that the intervention is so underused and, rather than to point at others (not available, patients refuse, not properly funded), what can we do to do better or initiate change?

I've thought about this a lot. Why is rehabilitation underutilised in comparison to other COPD therapies? It certainly isn't because it is less effective. Incontestable evidence shows that the improvements in exercise tolerance, dyspnoea and health-related quality of life are greater with pulmonary rehabilitation than any other COPD therapy. It isn't because it is more expensive, costs are modest compared with pharmacologic therapy. I think that our major failing is in advocacy and lobbying. The pharmaceutical industry, bless its soul, pours great energy and funding into lobbying regulators, legislators and third-party payors in support of their effective products. Pulmonary rehabilitation simply cannot match this! Pulmonary rehabilitation advocates have been wonderful at publishing high-quality guidelines documents and policy statements in prestigious journals, but I'm afraid they are not reaching the people we need to influence.

Is there a role to have rehabilitation more prominently present in study curricula of medical doctors or specialists in training? Today the possible benefits of rehabilitation for patients with lung disease or other chronic conditions do not receive much attention in the study trajectory of our "next generation" doctors.

Certainly, that would be an important step, but I don't think it will be enough. Just having physicians wanting it is not going to get the job done. We have to get payors to pay for rehabilitation, we have to have a cohort of rehabilitation experts trained to deliver rehabilitation. Currently such education is missing (at least in the USA) for rehabilitation professionals and we have to convince patients that they want to go to the programme. It's a whole bunch of things that need to come together.

\section{Pharmacotherapy (with drugs targeting bronchodilation, or perhaps the muscles) and pulmonary rehabilitation go, in my opinion, hand in hand. How do you see the interplay, and is there more to gain looking forward?}

Well, here is another point where physiology holds the answer. It is now clear that dynamic hyperinflation limits exercise tolerance in COPD patients with a ventilatory limitation. Dynamic hyperinflation at a given level of exercise can be reduced in one of two ways: provide more time for exhalation or speed the rate of exhalation. Rehabilitative exercise does the former and bronchodilators the latter. It's been well shown that these approaches to reduce dynamic hyperinflation are additive [8], so it is to be expected that optimal bronchodilation will amplify the effectiveness of rehabilitative exercise.

Studies of pharmacologic agents targeting the muscles have yielded a mixed bag. Some studies of anabolic steroids have shown modest improvements in muscle strength but, in general, it can be said that the best way to improve muscle function is, by far, rehabilitative exercise training. Further research with new molecules is worth pursuing though.

What are, in your opinion, the most important challenges moving forward for further advancing treatment for COPD in general?

Being honest, we've been remarkably unlucky in finding new mechanism drugs that benefit COPD patients. Drugs that either slow disease progression or decrease mortality have been introduced to treat many of the chronic diseases that plague humanity, but not COPD. We can only hope that drugs emerge 
from the basic research laboratories that we can evaluate in clinical trials. Hope springs eternal!

\section{Similarly, what is the biggest challenge for research in pulmonary rehabilitation apart from its practical application? What study are we still missing?}

For a long time, l've felt that, if we had a welldesigned clinical trial convincingly demonstrating that pulmonary rehabilitation prolongs survival in COPD, it would be a game changer. Therapies that improve survival are high in the pantheon of priorities for patient care. Such a study would, l'd assert, lead to reformulation of health policy, resulting in improved access and uptake of pulmonary rehabilitation. Obtaining funding for this clinical trial has proven to be exceedingly difficult though. Without a doubt it would be an expensive, time-consuming and difficult to conduct study. Recently, what I consider to be "second best" (but pretty good!) evidence has emerged. Peter Lindenauer's group has conducted a large Medicare database interrogation and found that those patients who received pulmonary rehabilitation after a COPD hospitalisation had better 1-year survival than those who didn't [9]. I have to say that these data concur with what I believe in my heart of hearts: patients who receive rehabilitation do better and live longer.

\section{So with this trial at hand, are we done with rehabilitation research (if it can be replicated) or are there other open questions?}

Far from it! First, there are limitations to these medical records studies. We can't be sure that there aren't unmeasured confounders that interfere with our interpretation. For example, in the excellent study by Lindenauer [9], there is no data on lung function, only that the subject carries the diagnosis of COPD in the medical record. Rehabilitation has been saddled with the inability to organise large studies to answer important questions (unlike our colleagues from the pharmaceutical industry), so there is plenty of work to do for young talented rehabilitation scientists.

\section{What would be, in your opinion, a good mission statement for the next decade in pulmonary rehabilitation. Where do we need to be in 10 years?}

The top three priorities are: 1) access, 2) access, and 3) access. Improved access will require a fair reimbursement for this therapy by governmental agencies and third-party payors. It will also require training of a new generation of healthcare providers, trained in multiple disciplines, to deliver rehabilitative therapy. Physicians, not only pulmonologists, but primary care physicians as well, will need to be convinced to refer their symptomatic COPD patients to rehabilitation. Finally, we need to do a better job in convincing patients to initiate, and stick with, their programme of rehabilitation. A lot of work to do!

\section{I like your three priorities. Should patients help us to achieve that goal? And, if so, how can we help patients to get that message across?}

This is an all-hands-on-deck process. Healthcare providers, researchers, payors and patients will all be important. Educating our COPD patients is important. An example of where this is being done is the 360 Social program of the COPD Foundation, which has reached 30000 or 40000 patients, even though this is still only a small fraction of the COPD population.

\footnotetext{
Affiliations

Thierry Troosters ${ }^{1}$, Richard Casaburi²

${ }^{1}$ Dept of Rehabilition Sciences, KU Leuven, Leuven, Belgium and Pulmonary rehabilitation, University Hospitals Leuven, Leuven, Belgium. ${ }^{2}$ Rehabilitation Clinical Trials Center, Lundquist Institute for Biomedical Innovation, Harbor-UCLA Medical Center, Torrance, CA, USA.
}

This article has supplementary material available from breathe.ersjournals.com

Conflict of interest

T. Troosters was president of the European Respiratory Society and reports grants and speaker fees paid to his institute from Boehringer Ingelheim, and speaker fees paid to his institute from Chiesi (BEL), in the past 3 years. R. Casaburi has nothing to disclose. 


\section{References}

1. Casaburi R, Petty TL. Principles and Practice of Pulmonary Rehabilitation. Philadelphia, Saunders, 1993.

2. Casaburi R, Patessio A, loli F, et al. Reductions in exercise lactic acidosis and ventilation as a result of exercise training in patients with obstructive lung disease. Am Rev Respir Dis 1991; 143: 9-18.

3. Maltais F, LeBlanc P, Simard C, et al. Skeletal muscle adaptation to endurance training in patients with chronic obstructive pulmonary disease. Am J Respir Crit Care Med 1996; 154: 442-447.

4. Casaburi R, Storer TW, Wasserman K. Mediation of reduced ventilatory response to exercise after endurance training. J Appl Physiol (1985) 1987; 63: 1533-1538.

5. Garcia-Aymerich J, Lange P, Benet M, et al. Regular physical activity reduces hospital admission and mortality in chronic obstructive pulmonary disease: a population based cohort study. Thorax 2006; 61: 772-778.
6. Rutkowski S, Rutkowska A, Kiper $\mathrm{P}$, et al. Virtual reality rehabilitation in patients with chronic obstructive pulmonary disease: a randomized controlled trial. Int J Chron Obstruct Pulmon Dis 2020; 15: 117-124

7. Casaburi R. Whither pulmonary rehabilitation? Will alternative modes help or hurt? Eur Respir J 2018; 52: 1801678.

8. Casaburi R, Kukafka D, Cooper CB, et al. Improvement in exercise tolerance with the combination of tiotropium and pulmonary rehabilitation in patients with COPD. Chest 2005; 127: 809-817.

9. Lindenauer PK, Stefan MS, Pekow PS, et al. Association between initiation of pulmonary rehabilitation after hospitalization for COPD and 1-year survival among Medicare beneficiaries. JAMA 2020; 323: 1813-1823. 